\title{
Stimulus control by part of a complex $\mathbf{S}^{\Delta}$
}

DAVID A. ECKERMAN

UNIVERSITY OF NORTH CAROLINA 1

Pigeons were trained to peck at each of four stimuli projected onto a response key. Subsequently, responding was reinforced in the presence of a compound stimulus composed of two of these stimuli (red background with horizontal white bar) and extinguished in the presence of a.compound stimulus composed of the other two stimuli (green background with vertical white bar). In an extinction session following this discrimination training, each of the four stimuli were again presented separately. Differential responding was found to the components associated with reinforcement and to the components associated with nonreinforcement. These results replicate earlier demonstrations of selective control by part of a complex $S^{D}$ and add a demonstration of selective control by part of a complex $S \Delta$.

Selective control of responding by one dimension of a complex discriminative stimulus $\left(\mathrm{SD}^{\mathrm{D}}\right)$ has been demonstrated in several studies (e.g., Reynolds, 1961; Lashley, 1942; Sutherland \& Mackintosh, 1964). As of this date, however, I know of no demonstration of the selective control of non-responding by one dimension of a complex $\mathrm{S}^{\Delta}$. There is at least one example of nonselective control. In the Reynold's (1961) experiment demonstrating responding to only one component of the $\mathrm{s}^{\mathrm{D}}$, neither component of the $\mathrm{s}^{\Delta}$ obtained significant amount of responding. Since no measure of the strength of response to each of the components was made prior to discrimination training, however, it is impossible to assess this lack of response strength to either component of $S^{\Delta}$. It is not, for instance, possible to determine whether this nonselective control reflects a reduction of response strength in both components or the mere absence of response strength to these two stimuli. In the present experiment, response strength was first increased to each of four component stimuli prior to discrimination training involving one pair as $\mathrm{S}^{D}$ and the other pair as $s^{\Delta}$. Results of an extinction test in which each of the components was again presented separately offer evidence of the selectivity of control both by $\mathrm{S}^{\mathrm{D}}$ and $\mathrm{S}^{\Delta}$ stimuli.

\section{Subjects}

Three 4 to 6-year-old male white Carneaux pigeons without prior experimental history were maintained at $80 \%$ of free-feeding weight.

\section{Apparatus}

A single-key (1 in. diameter) pigeon chamber having an IEE stimulus display cell with No. 1820 GE bulbs and a feeder, which presented a seed mixture $(40 \%$ vetch, 50\% kaffir corn, $10 \%$ hemp) for 3 sec. periods.

\section{Procedure}

Initial training. Key-pecking was first established to each of four stimuli projected onto the response key-green, red (colors fllling the key); white vertical line, white horizontal line (lines on dark achromatic background). Twenty-five 1-hr. sessions were given in which responses to each of these stimuli were reinforced according to a random interval schedule of reinforcement with a mean expected inter-reinforcement interval of $1 \mathrm{~min}$. (RI 1). In this schedule $\mathrm{T}=6$ sec., $\overline{\mathrm{T}}=1$, and $\mathrm{P}=.10$ (Farmer, 1963). Stimuli were changed once per min. according to a scrambled order which equated the number of presentations of the four stimuli over sessions. Stimulus periods were not separated by blackouts.

Discrimination training. Stimuli were then paired in the following manner: red background with white horizontal line (RH) versus green background with white vertical line (GV). RH then became the $\mathrm{S}^{\Delta}$ and $\mathrm{GV}$ the $\mathrm{SD}$ in a multiple $\mathrm{RI} 1$ extinction schedule of reinforcement. Training was given for $101 \mathrm{hr}$. sessions. Stimuli changed once per min. in these segsions, according to a scrambled sequence.

Test for stimulus control. Two $1 \mathrm{hr}$. sessions were given in which each of the four component stimuli was presented 15 times for $1 \mathrm{~min}$. periods. These sessions included no feeder presentations.

Results

Initial training. Although "color preference" waxed and waned over the 25 sessions of initial training to each of the four stimuli (see Morse \& Skinner, 1957), these preferences did not lead to more than $36 \%$ of the responses being emitted to any one of the four stimuli on any given day. Both "preferences" and "avoidances" were noted, but no particular stimulus was consistently either preferred or avoided either across birds or across more than three sessions for a particular bird.

Over $98 \%$ of responses were emitted to the $\mathrm{S}^{\mathrm{D}}$ pair on days 9 and 10 of discrimination training. Initial levels of accuracy in the discrimination training could be accurately predicted from the preferences and avoidances observed on the day before discrimination training started.

Test for stimulus control. Data for this phase of the experiment are presented in Figs. 1 and 2. Fig. 1 contains bar graph representations of the percent of responses obtained in the test emitted in the presence of each of the four stimuli. Fig. 2 presents free-hand cumulative records for responding in the 30 presentations of each of the four stimuli. Data show a selective control by one of the $\mathrm{SD}$ components for birds 2 and 4. Note that this selective control was obtained even though response strength had been initially equated for the two $\mathrm{S}^{D}$ components.

Selective control of non-responding was also ob- 
served. For bird 4, a greater percentage of responding was observed to one of the two $\mathrm{s}^{\Delta}$ components. The predominant $\mathrm{S}^{\Delta}$ component $(\mathrm{H})$ obtained even more responding than one of the $\mathrm{S}^{\mathrm{D}}$ components $(V)$. For this bird, then, there is strong evidence that the effect of earlier extinction had been to reduce response strength to only part of the compound $\mathrm{s}^{\Delta}$. Histograms for the other two birds do not reveal evidence for a selective control by part of the compound $\mathrm{s}^{\Delta}$. The cumulative response curves in Fig. 2, however, reveal an interesting pattern of changes within the test which may be related to selective control. For bird 3, for example, the extinction process differed markedly for the $H$ and $R$ components. Nearly 200 responses were emitted to $H$ on test day 1 while only 50 were emitted to $R$. Such a distribution of responses represented a selective control of nonresponding by $R$. On test day 2 , however, $\mathrm{H}$ obtained only 50 responses while $R$ obtained 120 . The control was reversed, then, in this second test. We can no longer designate the control as selective, but certainly the two components exhibit an independence of control. This independence itself is part of the phenomenon of selective control, showing such a phenomenon to exist even where the total number of responses emitted during 30 presentations do not

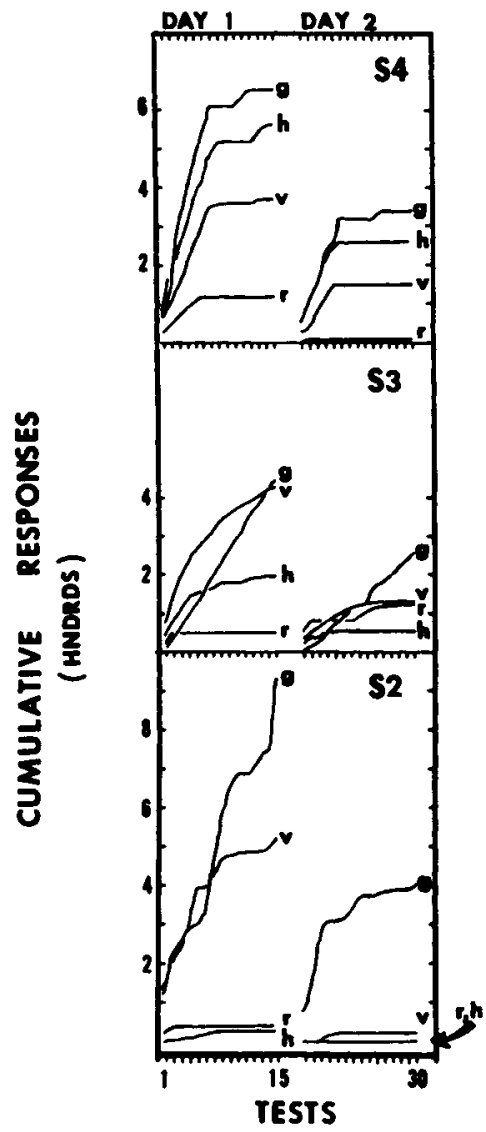

Fig. 2. Free-hand cumulative response records for responding to each of the stimuli during two days of extinction testing. Bird numbers are given in the upper right of each panel.

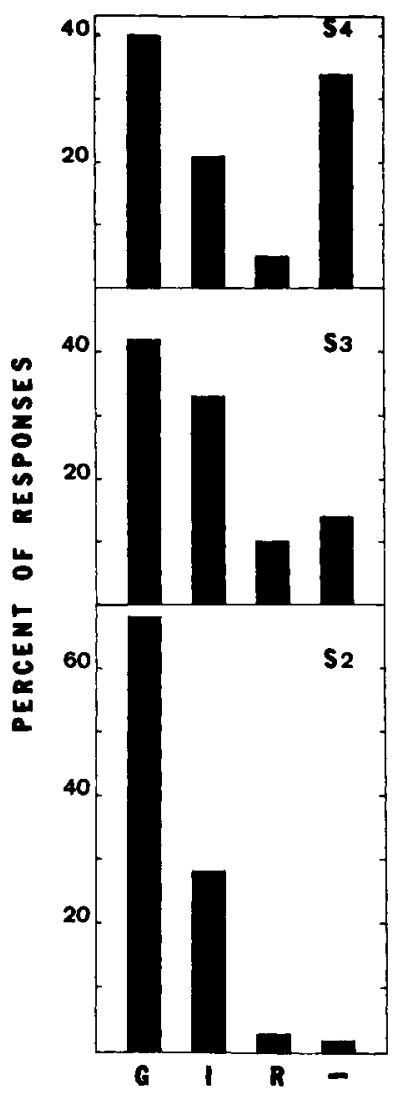

Fig. 1. Percent of responding to each of the four stimuli during extinction testing.

differ markedly. A similar independence of effect was observed for bird 2. On test day 1 responses were obtained to $R$ on the first presentation only, while responding was maintained over a much longer time to $\mathrm{H}$.

For all birds there is, then, evidence for an independence of effect for the two $\mathrm{s}^{\Delta}$ components. For bird 4 this independence is clearly of a variety which might be termed selective control by part of the complex $\mathrm{S}^{\Delta}$.

\section{References}

Farmer, J. Properties of behavior under random-interval reinforce ment schedules. J. exp. Anal. Beh., 1963, 6, 607-616.

Lashley, K. S. An examination of the continuity theory as applied to discriminative learning. J. gen. Psychol., 1942, 26, 241-265.

Reynolds, G. S. Attention in the pigeon. J. exp. Anal. Behav., $1961,4,203-208$.

Morse, W. H., \& Skinner, B. F. A second type of "superstition" in the pigeon. Amer. J. Psychol., 1957, 70, 308-311.

Sutherland, N. S., Mackintosh, N. J. Discrimination learning: non-additivity of cues. Nature, 1964, 201, 528-530.

\section{Note}

1. This work was supported by USPHS grant MH-10384 from the National Institute of Mental Health and by University Research Council grant 324-ALU-1(477) from the University of North Carolina. Reprints available from the author, Department of Psychology, University of North Carolina, Chapel Hill, North Carolina 27514. 